\title{
La gerencia, el rediseño de la empresa y las comunicaciones
}

\author{
Carlos FREUNDT CRUZ
}

\section{INTRODUCCIÓN}

La necesidad de desarrollar formas para que las entidades sean dirigidas de modo cada vez más eficiente hacia el logro de sus fines, ha generado un conjunto de conocimientos que los académicos han recopilado metódicamente con el fin de analizarlos, evaluarlos y utilizarlos didácticamente en la formación de aquéllos que deben asumir la responsabilidad de dirigir entidades en períodos más cortos de lo que se logra con el tiempo y la experiencia.

El mundo ha venido avanzando a través de una serie de procesos desencadenados por el desarrollo de la tecnología, la búsqueda de la satisfacción de las necesidades sociales y económicas de la población del mundo, y los esquemas de dirección política escogidos por los gobiernos con la mejor intención de saciar las necesidades del bien común.

Los países industrializados de Occidente perdieron preeminencia económica al surgir en el Oriente nuevos países con potencial económico y comercial, lo que ocasionó que los países busquen aliarse para hacer frente a una cada vez mayor competitividad mundial y agresividad por copar nuevos mercados.

Tanto los académicos dedicados al estudio de la empresa, como los profesionales dentro de éstas, tienen que permanecer en la búsqueda de nuevas maneras de conducir operaciones, para que el elemento humano sea capaz de enfrentar los nuevos cambios y necesidades. Han venido evolucionando las características y condiciones bajo las que se ha 
estudiado al ser humano a través de los años, con el afán de buscar una mejor dirección y resultados en la gestión de las empresas. En este proceso el comunicador organizacional asume gran importancia profesional, porque en todo momento el hombre ha sido el sujeto relevante del estudio; unas veces bajo un concepto mecanicista, otras teniendo presente su personalidad y su cultura, posteriormente evaluando su rol directivo y, más recientemente, actuando en equipos multivalentes de personas, cuya interacción concurre no solamente a la aplicación de un conocimiento sino a la transmisión de los valores propios de cada institución y de la relación y comunicación con su entorno.

\section{DE LA GERENCIA TRADICIONAL A LA GERENCIA ESTRATÉGICA}

Con el propósito de que el comunicador organizacional ubique su rol nos referiremos a dos libros que abarcan el desarrollo de las ciencias administrativas o ciencias de la empresa. El primero de ellos es Introducción a la teoría general de la administración, del autor brasileño Idalberto Chiavenatto. El otro, Administración y estrategia, pertenece a los argentinos Hermidia, Serra y Katiska.

Intencionalmente sustituiremos la palabra administración por la de gerencia en el transcurso de esta exposición, dado que, según nuestra interpretación, la acepción administrar conlleva un sentido más pasivo que el de gerenciar. En el primero de los casos se remarca la actuación en función de lo decidido o dispuesto por otros estamentos superiores. En cambio, el concepto moderno de gerencia incluye el respeto por las normas y decisiones superiores; pero en su contexto estratégico significa fomentar la formación de la actitud empresarial del gerente. No debe ser sólo un servidor dócil y disciplinado, más bien debe tener la característica de entrepreneur, debe estar informado y saber comunicarse eficientemente con los públicos internos $\mathrm{y}$ externos y es aquí donde la dirección de empresas y entidades exige un tratamiento profesional de la comunicación.

\section{Gerencia tradicional}

Previa a la obra de Taylor Los principios de la gerencia científica (1911) y la de Fayol, Gerencia industrial y general (1916), los académicos de las ciencias administrativas, entre los que se puede mencionar a Claude S. George Historia del 
pensamiento administrativo, encontraron en diferentes eventos del desarrollo de la civilización algunos aspectos relacionados con la gerencia. Así, por ejemplo, se menciona que los egipcios en el año 400 a.C. ya trataban los conceptos de planear, organizar y controlar. El código babilonio del año 1800 a.C. se ocupaba de los testimonios escritos y del salario mínimo y establecía que la responsabilidad no debía ser transferida al subalterno. Siglos después las obras de Nicolás Maquiavello, Adam Smith, Charles Babbage y otros trataron aspectos relacionados con la organización, el liderazgo, la especialización de los trabajadores, el estudio del tiempo y el movimiento, el concepto de costos y otros semejantes.

Idalberto Chiavenato en su interesante recopilación de conceptos, que provienen de más de 200 obras escritas desde 1903 hasta 1972, los agrupa por tendencias de acuerdo con esta cronología:

\begin{tabular}{ll}
\hline AÑOS & TEORÍAS \\
\hline 1903 & Administración científica \\
1909 & Burocrática \\
1916 & Clásica \\
1932 & De las relaciones humanas \\
1947 & Estructuralista \\
1951 & De sistemas \\
1953 & Enfoque sociotécnico \\
1954 & Neoclásica \\
1957 & Del comportamiento \\
1962 & Desarrollo organizacional \\
1972 & Contingencial \\
\hline
\end{tabular}

Para hacer una breve descripción de los aportes y contenidos de estas teorias, las clasificaremos en cuatro grandes grupos:

a) Teorias sobre funciones, estructura y gerencia: Administración científica (1903), Burocrática (1909), Clásica (1916), Estructuralista (1947), Enfoque sociotécnico (1953), Neoclásica (1954).

b) Teorias basadas en las personas: De las relaciones humanas (1932), Neoclásica (1954), Del comportamiento (1957), Desarrollo organizacional (1962).

c) Teoria de sistemas: la cibernética, la informática y el uso de matrices matemáticas en la administración (1951). 
d) Teoría de la contingencia (1972): la relación de la empresa con sus ambientes. La evaluación de los aspectos situacionales internos de la organización para adaptar su estilo de gerencia a las necesidades del momento y del grupo.

En cada grupo consideraremos, de igual manera, conceptos de los nueve primeros capítulos de la obra de Jorge Hermida, Roberto Serra y Eduardo Katiska.

\subsection{Teorias sobre funciones, estructuras y gerencia}

Taylor aplica el método científico para analizar las acciones que el hombre mecánicamente desarrolla para cumplir una tarea. Debe reconocerse esto como el inicio de la ingenieria humana. Ésta se desarrolla buscando la adecuación del cuerpo humano a la conducción de vehículos, incluidos aquéllos con los que se han realizado vuelos espaciales. La contribución de Taylor también comprendió la importancia que debía darse a la relación directivo-subordinado, así como el suministro de pautas para la selección y capacitación de personal.

Fayol, por su parte, estudió las organizaciones y su operatividad, y contribuyó a definir los elementos que componen la tarea de la gerencia. Agrupó los diferentes tipos de actividades que se dan en las empresas estudiadas en razón de seis grandes funciones:

a) Técnicas, relacionadas con la producción de bienes o de servicios.

b) Comerciales, relacionadas con la compra, la venta o el intercambio.

c) Financieras, relacionadas con la búsqueda y gerencia de capitales.

d) Seguridad, relacionadas con la protección y preservación de los bienes de las personas.

e) Contables, relacionadas con los registros de los hechos ocurridos en la empresa, inventarios, estadisticas y la función administrativa.

f) De gobierno o gerencia, aquéllas que coordinan y sincronizan las demás funciones de la empresa.

En cuanto a la función gerencial, Fayol distingue cinco partes del proceso a las que denomina: previsión, organización, dirección, coordinación y control. Cada una de estas actividades realizadas por aquéllos que tienen que dirigir a otros subordinados, en el cumplimiento de las funciones específicas mencionadas en la relación de las cinco funciones anteriores. 
Cabe mencionar que años atrás, en 1945, la Sociedad Americana de Ingenieros Mecánicos, después de realizar en los Estados Unidos un estudio similar al de Fayol, clasificó las actividades de la empresa con denominaciones similares, incluyendo las funciones de investigación y desarrollo y de control interno, y separando la dirección de recursos humanos de la de administración. De este modo permanecen las funciones: financiera, de producción y comercial.

Actualmente en nuestro país y especialmente en la Facultad de Ciencias Administrativas de la Universidad de Lima, clasificamos estas funciones específicas en el Área de Comercialización que comprende: Marketing y Ventas; Administración de Operaciones, que a su vez comprende Investigación y Desarrollo, Ingeniería de Planta, Producción y Logística; Administración Financiera, que considera todas las asignaturas de Economía, Contabilidad, Costos, Proyecciones, Riesgos y Seguros; Recursos Humanos, con las asignaturas de Dirección de los Recursos Humanos, Psicología Organizacional, Desarrollo Organizacional y Aspectos Legales y Laborales. Desde los primeros niveles se busca instruir al educando sobre la relación de estas áreas en procesos integrales, por medio de los elementos que conforman el planeamiento, la organización, la dirección y el control.

Opinamos que Fayol consideró como vital e importante al elemento humano en la función gerencial. Quizá por la época en que vivía asignó un grado menor de independencia en la toma de decisiones y un grado mayor de respeto a la autoridad. Esta preocupación lo llevó a establecer catorce principios, entre los que no dio suficiente énfasis a la comunicación.

En el esquema burocrático se trató de hacer de la definición de tareas, autoridad y relaciones algo más estable y permanente. Como escribía Fayol, para tener un "orden social" es necesario establecer un "orden material". Las estructuras de organización, desarrolladas por los neoclásicos incrementando las normas y métodos, sirvieron para diseñar las estructuras de organización de entidades cada vez más amplias, en que la planificación y administración de la comunicación se fue haciendo indispensable como actividad profesional, ya que incluso las relaciones traspasan fronteras nacionales y regionales, llegándose en diferentes partes del globo a las empresas internacionales y multinacionales con órganos de asesoría, de línea o de ejecución, apoyo y supervisión.

Surgió así el concepto de corporación, de funciones operativas internas e integrales, de interpelación entre em- 
presas. El desarrollo de nuevas normas y principios permitieron, gracias al desarrollo de las comunicaciones, aplicar las ciencias administrativas a diferentes grupos humanos ubicados en entidades de diferentes latitudes.

En el transcurso del tiempo se reconoció la necesidad del planeamiento y control como funciones básicas de la gerencia. Era necesario buscar principios que permitieran aglutinar los esfuerzos, intereses y actitudes de los elementos que conforman las organizaciones, es decir, mejorar la dirección. Éste fue uno de los principales aportes de los neoclásicos. No obstante, por la misma naturaleza social de las ciencias administrativas, no hay una aceptación universal de un conjunto de principios de dirección relacionados económicamente que complementen la función de la gerencia de planear, organizar y controlar.

Guiándonos por los principios de administración de Fayol, por la contribución de Simón y Mooney y por la de Antonio Pinilla Sánchez Concha en su libro Administración, ciencia de la acción directriz, modestamente intentamos establecer que lo escrito por diferentes autores, en cuanto a principios de dirección, puede reunirse en estas cuatro grandes características:

a) Los principios que se refieren o se relacionan con el objetivo.

b) Los principios que están referidos a la autoridad.

c) Los principios que están relacionados con los roles, tareas y actividades que desarrollan los elementos del grupo.

d) Los principios que tienen que hacer con la comunicación, entendiendo ésta en su forma más amplia; es decir, no sólo aquellos aspectos referidos al mensaje, codificación, decodificación, etc., sino también a la intención de comprender escuchando, de colaborar, de cooperar, de comprometerse con el cumplimiento de estos principios.

Esta enumeración sirve como recordatorio en el caso de que se tengan que dar pautas sobre tareas que deben ser realizadas por un número pequeño de personas.

En el caso de muchas personas o varios grupos se recomienda definir y aplicar los siguientes aspectos:

a) Establecer normas y directivas generales, así como niveles y canales de autoridad y sistemas integrales de comunicación.

b) Definir participativamente objetivos y funciones de las unidades de organización y puestos de trabajo y comunicarlos adecuadamente. 
c) Entrenar y fomentar la aplicación de la autoridad con idoneidad.

d) Incentivar y asegurar la máxima integración, colaboración y cooperación del personal en forma franca y abierta.

e) Involucrar al personal en el cumplimiento de los objetivos de la producción y esforzarse por lograr su productividad y eficiencia.

f) Estudiar, evaluar y cumplir con la justicia distributiva.

Los neoclásicos y también los estructuralistas tuvieron presente los conceptos de la organización informal, es decir, reconocian que las estructuras de organización no correspondian en la práctica a las relaciones y comunicaciones que se daban realmente en la operatividad de las empresas por la existencia de las relaciones personales.

\subsection{Teorias basadas en las personas}

En su deseo de buscar una mayor productividad del ser humano un grupo importante de autores detecta implicancias para el rendimiento en la conducta individual y grupal.

Esto llevó a los psicólogos y sociólogos a estudiar con mayor profundidad esta problemática, presentándose casos como el de la Universidad de Cornell, en Ithaca, Nueva York, en que se da un mayor peso en el currículo del estudiante de Gerencia a los aspectos relacionados con los recursos humanos. Surgen en esta universidad y en otras como la del estado de Ohio, conceptos referidos a la motivación del personal a través de las contribuciones de Maslow, Herzberg, McGregor, Argyris, Skinner y también en relación con los estilos de liderazgo, como en los escritos de Tannenbaun, Blake y Mounton, Reddin, y, finalmente, sobre la organización y sus funciones en Barnard, Simón, Skinner, Likert, etc.

En sentido estricto, estos profesionales condujeron estudios propios de su especialidad con métodos científicos, y las facultades de Ciencias Administrativas y de Ciencias de la Comunicación fueron exponiendo dichos conocimientos a sus alumnos, contribuyendo de este modo a que el estudiante reflexionara tanto sobre los aspectos propios de su personalidad en la empresa, como sobre las actitudes de sus dirigidos, iguales y superiores, y, en general, sobre los aspectos de interacción y comunicación.

Con la contribución de autores como Schein, Bennis y Bekhard, y por el deseo de hacer de mayor transferencia los 
contenidos de la psicología organizacional comienza a hablarse de la personalidad de la organización y de la cultura organizacional. Surge el concepto de análisis transaccional en la obra de Erick Berne y la metodología del desarrollo organizacional como una forma de operar y trabajar el cambio, llegándose a desarrollar esquemas como la dirección por objetivos, en que comienza a flexibilizarse la toma de decisiones por la mejora en las comunicaciones y la correspondiente mayor confianza mutua entre subordinados y jefe.

A continuación, en forma sucinta, se señalan algunos aspectos que recomendamos observar y evaluar cuando se ingresa a desempeñar un trabajo en una organización:

\section{ASPECTOS DE OBSERVACIÓN Y EVALUACIÓN EN UN GRUPO}

Fines:

Normas:

Poder e influencia:

Cohesión:

Clima laboral:

Conflictos:

Comunicación:
¿Se han establecido?, ¿son claros, entendibles por todos?, ¿aceptados?, ¿compartidos?

¿Definidas?, ¿difundidas?, ¿son actualizadas?, ¿son respetadas?

¿Quién o quiénes lo ejercen?, ¿cuál es su razón de ser: amistad, vínculo familiar, carisma, conocimiento? ¿Existen subgrupos informales?, ¿se ha definido la autoridad formalmente?, ¿es aceptada?, ¿hay espíritu de cuerpo?

¿Existe libertad de opinión?, ¿se acepta la actitud critica?, ¿hay posibilidad de cambio?, ¿cómo se dan las relaciones internas?

¿Existen?, ¿quién o quiénes lo generan?, ¿cómo se resuelve?, ¿por escape?, ¿por enfrentamiento?, ¿las razones del conflicto son subjetivos u objetivas?, ¿hay deseo de solución?

¿Se da sobre la base de un sistema?, ¿es formal?, ¿informal?, ¿ambos?, ¿es de apertura? 


\subsection{Concepto de sistemas}

El enfoque de la teoría de sistemas en la administración puede considerarse dentro de tres grandes aspectos:

- La empresa como sistema social.

- La cibernética.

- El uso de matrices matemáticas en computadora y otras técnicas.

En cuanto al primer aspecto, cuando se inicia el estudio de las funciones de la empresa, se descompone este todo en sus diferentes partes, con el fin de analizar sus elementos y atributos y ver cómo es que se originan sus resultados. La obra de Fayol inicia este tipo de análisis.

Más adelante, al comprobarse el efecto del ambiente en las organizaciones, se reconoce a la empresa como parte de un sistema social mayor. Autores como Mooney, al establecer que en los grupos sociales hay funciones de tipo resolutivo, funciones de ejecución y funciones de interpretación e integración, están identificando al Poder Legislativo, al Poder Ejecutivo y al Poder Judicial, constitutivos de un Estado. Las funciones genéricas de las empresas podrian simbolizarse por estos tres tipos de funciones, correspondiendo al Directorio aquélla por la que se establecen disposiciones generales y normativas.

Aquellas funciones que se originan para el cumplimiento de las normas y acciones que coadyuvan al logro de los objetivos establecidos por el ente resolutivo superior (el directorio) son dirigidas por el gerente general, los gerentes de las áreas específicas y por todos los jefes y profesionales que a ellos reportan, a través de un sistema internamente relacionado.

La función que corresponderá al aspecto interpretativo y de integración en la entidad también le corresponde a cada gerente o cabeza de sistema y requiere del buen uso de las comunicaciones externas e internas de la empresa.

La empresa es una reunión de profesionales de diferente especialidad. Las evaluaciones específicas y recomendaciones que ellos plantean, se elevan a través de la gerencia general para ser puestas a consideración del directorio, pero integrándose con las recomendaciones de las otras gerencias, conformando programas de gestión participativos e integrales. Para facilitar esto se requiere el conocimiento especializado del profesional en comunicaciones.

Este conjunto de actividades constituye el Sistema de Dirección Integral del Sistema Empresa al cual pertenece el Sistema de Información Gerencial. Son muchos los autores 
que se ocupan de estos conceptos, considerando de principal importancia la obra de Fernando del Pozo Navarro Dirección por sistemas; otros autores que deben ser mencionados son L. von Bertanlanfy y F.E. Kast y Rosenzweig.

En lo que se refiere al aspecto cibernético y matemático día a día, tanto la robótica en los aspectos de automatización fabril, como la informática en el de tratamiento de la información ha vuelto masivo el uso de la computadora. Desempeñar un cargo de responsabilidad profesional o de directivo en la empresa requiere del uso de computadoras y saber acceder a información, porque facilita el estudio de alternativas y la toma de decisiones. En lo que se refiere al uso de las matemáticas y la computadora podemos mencionar que se ha ido sofisticando el uso de matrices y modelos para el tratamiento de la data numérica, lo que permite por ejemplo hacer estudios de factibilidad e investigación de operaciones.

Todo esto permite analizar y desarrollar vias alternativas de acción y continuar el proceso de realizar las variaciones en forma proyectada, logrando de este modo soluciones alternativas de gran impacto y bajo costo.

\subsection{Teoria de la contingencia}

Consideramos que la inclusión de esta teoría en las últimas ediciones del libro de Chiavenato ha servido para reforzar los conceptos del autor ante una tendencia inadecuada de algunos de sus lectores, que al utilizar su libro como material didáctico o texto de consulta entendian que la empresa debía dirigirse de acuerdo con alguno de los enfoques específicos que en dicha obra se enunciaban.

El enfoque contingencial considera la necesidad de que las organizaciones se vayan ajustando a los cambios del ambiente, sean éstos por influencia de la economía global en los diferentes países, como también por el macroambiente propio del país en el que opera la empresa, considerando los aspectos tecnológicos, culturales, ecológicos, demográficos, sociales, económicos, políticos y legales, así como los del microambiente, o sea la influencia más directa de los clientes, los proveedores, las entidades financieras, la competencia, etc.

El concepto de contingencia tomado dentro del marco laboral relacionado con la formación de ejecutivos enseña, además, la importancia de conocer la tendencia personal en cuanto a estilo de liderazgo, el mismo que debe adecuarse a 
las características específicas de cada momento, de la capacidad de los grupos que dirigimos y de sus actitudes.

Es necesario estar informado permanentemente y conocer los diferentes enfoques con el fin de encontrar la adecuación ideal de cada uno de ellos a las situaciones cambiantes y hacer los ajustes buscando la eficiencia de las organizaciones.

\section{Gerencia estratégica}

En realidad ya se han adelantado algunos conceptos de lo que los autores Hermida, Serra y Katiska llaman la "gerencia estratégica". La teoría de la contingencia preparó el estado del arte para entender las nuevas denominaciones o modos de la gerencia.

Desde mediados de la década de los sesenta se dan cambios en el mundo en los aspectos tecnológicos y económicos que influyen en los estilos de dirección de las empresas y en su manera de afrontar su proyección en el tiempo y en la amplitud de cobertura de sus operaciones.

Los especialistas sostienen que contribuyeron a esos cambios la reducción del auge comercial de los Estados Unidos y algunos países europeos, la pérdida de hegemonía militar de los ejércitos americanos, la problemática del petrodólar, los cambios de regímenes de gobierno en lo económico-político, el surgimiento de potencias comerciales asiáticas y las reacciones sociales en varios países del Tercer Mundo.

Los mercados tradicionales que venían creciendo llegaron a lo que se denominó "mercados estancados". Esto creó un problema de alta competitividad a los países industrializados que no previeron el cambio de los mercados permanentemente crecientes. Los mercados se volvieron inestables y se creó la llamada "turbulencia". Es decir, los factores que tradicionalmente se consideraban posibles de evaluar para proyectar las actividades de las empresas, ya no pueden ser pronosticados con facilidad. Sufre la economía de los países tradicionalmente fuertes y afecta las economías y las empresas de los países de menor auge comercial o fabril. Se consideró que todo lo hecho en las ciencias de la empresa debió ser revisado y se habló del cambio de paradigmas en la gerencia.

Aparecen los gurús como Naisbitt, con sus megatendencias, y Toffler, con su simbología de las olas, relacionándola con el avance socioeconómico de la civilización. La Primera Ola se caracterizó por la facilidad en la tenencia de 
las tierras. Se contaba con mano de obra barata, en algunos casos mediante la esclavitud. Esta ola se caracteriza por ser dependiente de la naturaleza, por su estrecha relación con el agro.

A partir de 1900 sigue la industrial mecánica, la Segunda Ola, en que las materias primas y los salarios son baratos. Muchos de los países del Tercer Mundo se encuentran en esta etapa. Algunos tratan de ingresar a la Tercera Ola que se caracteriza por el uso de la electrónica y la información, el acceso barato al conocimiento y una mayor independencia de la naturaleza.

Otro prolífico profesional académico y autor de esta época moderna es Peter Drucker. Ha escrito, en base a sus investigaciones, experiencias y proyecciones, más de 15 obras que también son clásicas en la gerencia. Su preocupación estuvo siempre dirigida a salirse de los marcos tradicionales, tanto para la dirección de la empresa como para desarrollar sus planes. Recomienda la actitud emprendedora, renovadora y advierte sobre los cambios que se presentarán en las organizaciones. Sostiene que la tecnologia, la inversión y la capacidad del hombre, es lo que permite tener entidades actualizadas y competitivas.

Hermida, Serra y Katiska subdividen los nuevos esquemas o paradigmas en un sector duro (hard), que expresa mayor preocupación por lo técnico instrumental para el análisis y solución de los problemas de la empresa y de sus mercados frente a la turbulencia y otro sector denominado blando (soft), referido a la mayor participación de la persona, al fomento de su iniciativa y su creatividad, como una condición para lograr el éxito con los enfoques duros.

La cultura de la organización es considerada como un aspecto de vital importancia para asegurar la modernización constante. Ésta no es otra cosa que el conjunto de prácticas, costumbres y creencias de cada organización, como sostiene el autor chileno Fernando Flores en su libro Creando organizaciones para el futuro. Cada organización no es otra cosa que el conjunto de personas que pertenecen a ella por vínculos de interés, necesidad y realización; y su interacción se realiza por medio de comunicaciones.

Las nuevas tendencias de los tres autores mencionados se presentan de la siguiente manera en el libro Administración y estrategia: 
Planeamiento estratégico

1970

1980

1985

Modelos de análisis de
negocios

Estrategia competitiva

Ventajas competitivas
Globalización empresarial

Alianzas estratégicas

Ecología

\section{ESTRATEGIA \\ ESTRUCTURA \\ CULTURA}

Gerencia japonesa

Intentos de aplicación del estilo

japonés en empresas

occidentales

Búsqueda de la excelencia

Calidad total

Adecuación de las estructuras

Habilidad para la negociación

Entrepreneurship

Intrapeneurship

Dinámicas

Culturas compartidas

\subsection{Descripción de las caracteristicas saltantes de los nuevos paradigmas - Enfoque duro}

\section{a) El planeamiento y la gerencia estratégica}

Las funciones básicas de planeamiento, organización, dirección y control, siguen siendo la base de donde parten las decisiones, normas, comunicaciones, acciones, reportes y evaluaciones de las diferentes actividades que se cumplen en las empresas en sus áreas funcionales de: Comercialización, Administración de operaciones, Dirección de recursos humanos y Administración financiera, sean éstas locales, nacionales, internacionales o globales.

El planeamiento requiere de evaluaciones constantes, de definiciones, de objetivos, de establecimiento de pautas que permitan la toma de decisiones en diferentes lugares o niveles jerárquicos de la entidad. De igual manera requiere el diseño de programas, estrategias, presupuestos y procesos que se concreten y comuniquen en la organización.

La organización o, como se denomina ahora, "estructura" ayuda a establecer la ubicación de las funciones, procesos y procedimientos, que coadyuvan a establecer los sistemas de información gerencial. 
La dirección es ejercer la autoridad, comunicar, supervisar y evaluar el cumplimiento de acciones y resultados y determinar ajustes.

El control consiste en establecer las pautas o criterios para la evaluación y medida de acciones y resultados, y ejecutar los procesos de los sistemas de información gerencial que permitan ir modificando y adecuando acciones según los resultados obtenidos

El planeamiento como proceso ha evolucionado desde la preparación de presupuestos a corto plazo entre el contador de la empresa y el gerente general, para conocer las necesidades financieras, hasta etapas más sofisticadas en que se proyectaban rendimientos de flujos de caja, costos, precios y márgenes de contribución y proyecciones de rendimiento de inversión en el tiempo.

Actualmente el planeamiento es participativo, integrador y estratégico. Este planeamiento requiere de una diagnosis interna de la empresa hecha en forma integral y sistemática. Se toma en cuenta los factores de la influencia inmediata a la gestión de la empresa, como también aquéllos que la puedan afectar de una manera indirecta, tanto en lo que se refiere a los aspectos de macroentorno como a las influencias de las corporaciones globalizadas y las relaciones monetarias con organismos financieros internacionales.

Este planeamiento estratégico se desarrolla sobre la base del estudio constante del mercado, el desarrollo de programas y demás aspectos relacionados con la comercialización. El desarrollo y mejora de productos es otro aspecto vital que debe considerarse, como también los aspectos logísticos, productivos y de personal para llegar a programas integrales concretados numéricamente.

En otras palabras, las diferentes áreas de la empresa o entidad deben participar de este proceso estratégico que, como se ha dicho, considera aspectos internos y externos de la empresa, constituyéndose, en una actividad gerencial estratégica que requiere que las personas sepan comunicarse o cuenten con la ayuda profesional para lograr esto.

Como las empresas se constituyen en personas jurídicas y suelen pertenecer en nuestros países a un cierto grupo de accionistas, cuyas actividades giran alrededor de ciertos tipos de negocios, esta estrategia que involucra a las diferentes empresas, se conoce como "estrategia corporativa" y se requiere previamente definir la visión de la razón de ser de la corporación como la definición de su misión a futuro.

Cada persona jurídica desarrolla su estrategia acorde con la misión y objetivos generales corporativos. Para esto las 
gerencias funcionales específicas desarrollan sus propias estrategias y programas que deben cumplirse dentro de cada empresa en forma integral, a esto se le denomina "gerencia estratégica operativa".

Podría darse el caso, igualmente, de que las empresas del grupo económico realicen actividades que pertenecen a diferentes negocios. Cuando se identifica una de estas lineas de negocios, de manera independiente de las diferentes empresas, se denomina "unidad estratégica de negocios".

\section{b) La estrategia competitiva}

Michael Porter, en 1980, escribe su libro Estrategia competitiva. Este libro, en los diferentes esquemas de análisis de la situación de los negocios de las empresas llega a tener éxito en un momento en que era necesario para la empresa americana encontrar solución a su pérdida de mercado, no sólo a nivel internacional sino dentro de su propio territorio.

En 1985, Porter nuevamente vuelve a tener un éxito de librería con su libro Ventajas competitivas, enfatizando que la competitividad dependía de la agregación del valor a los productos, pero que esto fuera percibido como una ventaja por el consumidor adicional al precio pagado.

Los esquemas de análisis de Porter proporcionan referencias más amplias de análisis de lo que tradicionalmente acostumbraba la gerencia, y esto justamente tiene un correlato con esta necesidad de ver la entidad bajo un panorama mayor, que le permita tener una visión a futuro, definir una misión, un propósito y buscar entonces las estrategias y la forma de evaluación y estructuras que aseguren su logro en el tiempo. La última obra de Porter, de 1990, es La ventaja competitiva de las naciones. En ésta se aprecia nuevamente la evolución de los aspectos técnicos y estructurales hacia la búsqueda de la creatividad y participación del hombre y la gerencia.

\section{c) Globalización empresarial}

El concepto de globalización tiene que ver con la cobertura mundial de las operaciones desarrolladas por las entidades.

De la última obra de Porter se puede tomar su denominado "diamante" que sintetiza los aspectos que deben analizarse para definir la internacionalización de los nego- 
cios. No obstante, como sostiene George Yip en sus libros sobre estrategia y globalización, es conveniente que las empresas dentro de sus países busquen aplicar estos conceptos de competitividad y globalización empresarial para ganar experiencia y salir a competir con éxito en otros países. Ha ayudado a estos aspectos de globalización los adelantos en el transporte, las comunicaciones y la cibernética. Ahora se habla de que la globalización no es otra cosa que el reconocimiento de diferentes aldeas similares en las diferentes partes del mundo a las que se puede llegar con productos, si éstos son de buena calidad y se ofrecen a buenos costos o precios variados o, en todo caso, si son diferentes de otros.

La globalización empezó con el marketing y la llegada de marcas y productos que eran consumidos en diferentes partes del mundo. La globalización empresarial ha tenido que variar los conceptos bajo los cuales se establecen las políticas, programas y estrategias desde una central, asi como las patentes, las franquicias, formas de producción y manejo financiero.

La globalización también comprende el estudio de la unión de países en mercados comerciales, así como el de las organizaciones financieras internacionales, las inversiones, las adquisiciones, fusiones, establecimiento de holdings en diferentes partes del mundo, aplicándose la tecnología de la información de país a país.

Esta obra la escribió Porter después de un trabajo encomendado inicialmente por el presidente Reagan y le tomó diez años estudiar cien empresas internacionales y siete países. Como resultado de la obra enfatiza que es el hombre en realidad quien hace la empresa exitosa y puede crear la ventaja competitiva de un país.

2.2 Descripción de las características saltantes de los paradigmas - Enfoque blando

\section{Gerencia japonesa - valores - calidad total - clima organizacional}

El auge en el desarrollo económico de Japón hizo pensar que habia formas de gerencia diferentes que era necesario que fueran estudiadas. Se partió de la hipótesis de que si se cambiaban los estilos gerenciales de Occidente se tendría éxito, como venía ocurriendo en las empresas orientales. El resultado del estudio demostró que no existía en realidad 
una forma de gerencia diferente sino una forma de actuación, de conductas, valores, cultura, que no correspondian a los que desarrollaban los occidentales.

Los estudiosos reencontraron la importancia que demanda estudiar la manera de ser de los pueblos, cómo se comunican y cómo esto influye en la manera de conducir las organizaciones.

En cuanto a diferencia de valores se encuentra que el pueblo japonés es solidario. Sus relaciones se basan en el respeto y la sutileza. Debido a la escasez de recursos tienden al ahorro, a ser productivos. Su preocupación constante es ser educados y leales. La empresa, la entidad, se desarrolla alrededor de una población. Se piensa que el empleo debe ser perpetuo. Las decisiones se toman por participación, pero dentro de procesos que requieren de tiempo y protocolo. Esto debe conocerlo el comunicador para planear su diagnosis y diseños apropiados en los sistemas de comunicación. No obstante las diferencias, se trató de copiar alguno de estos aspectos. Paradójicamente los esquemas del doctor Deming sobre calidad total, aunque desarrollados en los Estados Unidos pudieron ser aplicados con éxito en el Japón.

El autor William Oucchi, en su libro Teoría $Z$, explica la forma de ser del japonés, de observar, de seguir al "Sensei", obedecerlo, aprender de él para ir mejorando paulatinamente. Esto los lleva a adquirir una maestría que posteriormente les permitirá mejorar los procesos y resultados.

Aunque los esquemas de una cultura no pueden ser trasladados exactamente a otras, surgió aquí la necesidad del estudio de la cultura de los pueblos, de las etnias, de los grupos humanos y sus comunicaciones, como aspectos que tenían que ser considerados para mejorar la dirección de empresas, más aún cuando las entidades globalizadas deben trabajar con personal en diferentes partes del mundo.

Aun en las empresas locales la cultura organizacional es considerada fundamental para buscar el cambio. La mejora se obstaculiza no solamente por la natural resistencia al cambio de parte del subordinado en aspectos mecanicistas. Adicionalmente hay que considerar actitudes, estados de ánimo, creatividad, deseo de realización y capacidad de tomar decisiones.

El ámbito de este tipo de influencia para el profesional abarca el estudio de las relaciones con otros de su mismo nivel jerárquico y diferentes especialidades, así como con los jefes, hasta llegar al nivel de los miembros del directorio y los propietarios. Los cambios muchas veces no logran realizarse por falta de habilidad para comunicar las nuevas estrategias 
incentivando una actitud hacia el cambio.

\section{EL REDISEÑO DE LA EMPRESA}

Nos referimos finalmente a las recomendaciones de la Compañía IBM, una de las grandes empresas de los Estados Unidos que decidió establecer una gerencia estratégica y rediseñar sus estructuras.

Una de las nuevas entidades de este grupo empresarial, que sigue brindando sus servicios de manera independiente, es el IBM Advanced Business Institute. En nuestro país, F. César Toscano, nacido en Uruguay, con vasta experiencia docente profesional y académica y Allen L. Morton, Jr. con similares pergaminos, vienen dictando seminarios para orientar a los interesados en mejorar sus empresas. Hasta 1996 ya han dictado diez de estos seminarios.

La idea del que decide seguir un curso de esta naturaleza en este instituto posiblemente está dirigida a conocer más sobre aquel otro nuevo paradigma de la gerencia moderna que se denomina "reingeniería". El seminario en mención, sin embargo, partiendo del hecho de que las empresas vienen buscando un mejoramiento continuo, han venido aplicando las diferentes recomendaciones dadas por académicos y profesionales de las ciencias de la empresa. Entonces enseña que para llegar a reestructurar adecuadamente la empresa se debe considerar no un solo método sino varios, tal como sigue:

- Importancia del cliente.

- La orientación a los procesos.

- El benchmarking.

- Los facilitadores de tecnología de información.

- La reingeniería.

- Cultura organizacional.

Pero todos estos métodos deben estar desde un principio ligados estrechamente con el estudio y cambio de la cultura organizacional y, por consiguiente, opinamos con la labor del comunicador organizacional. Esto es de vital importancia tanto en la diagnosis, como en el diseño de soluciones y con mayor razón en la implementación del cambio.

\section{Importancia del cliente}

Es un aspecto vital para la transformación estratégica de las empresas. Consiste en tratar de establecer en las organizaciones una mentalidad de calidad total. Como es sabido, 
esto va más allá del tradicional concepto del control de calidad en la producción del bien o servicio.

La excelencia, la búsqueda de anular los defectos personales y de la organización es un propósito que debe instalarse en la mente de todos los trabajadores para brindar el mejor servicio o producto al cliente. Las técnicas del desarrollo organizacional y reforzamiento psicológico en este tipo de programas ayuda a ello, pero sobre la base de una buena comunicación.

Cada vez más entidades extranjeras están buscando que los proveedores peruanos obtengan certificaciones de calidad total, cumplimiento de normas como ISO 9000, para aceptar los productos o servicios.

\section{Orientación a los procesos}

Se define proceso como un conjunto de actividades relacionadas lógicamente, que producen un resultado final específico para un cliente interno o externo.

Estos procesos proveen la perspectiva de cómo la empresa hace su negocio, cómo opera, y a la vez permite tener un entendimiento de las interrelaciones del negocio y define de manera clara los puntos de contacto con el cliente.

Sobre esta base se busca el mejoramiento continuo, adicionalmente ésta es la única forma que permite, de ser necesario, aplicar la reingeniería a la empresa.

Existe una estrecha relación entre la definición de los procesos de la empresa, la integración de las funciones de las diferentes áreas y el denominado análisis del modelo de Cadena de Valor de Porter.

\section{El benchmarking}

El benchmarking se define como un proceso continuo y sistemático que permite evaluar los productos, servicios y procesos de trabajo de organizaciones reconocidas como rivales competitivos, por tener las mejores prácticas o productos, con el fin de tomar su ejemplo, creativa e innovadoramente para mejorar la propia organización y los productos.

Para esto es necesario conocer los factores críticos de éxito del negocio. Preguntarse ¿qué es lo que no está funcionando y cómo deseamos funcionar? De igual manera debemos conocer cuáles son las quejas de nuestros clientes, qué problemas hemos identificado, dónde está la competencia y en qué podemos hacer las mejoras más importantes. 
Todo el personal debería colaborar en ubicar estas necesidades, por ello es necesario capacitarse. Planear estas actividades e indagar solamente por aquello en lo que la empresa estaría presta a competir.

\section{Los facilitadores de tecnología de información}

El avance de la cibernética, de la computación, del uso de redes, de Internet, permite a las empresas obtener mejoras de gran importancia en su operatividad, las que coadyuvan a la reducción de tiempo en la preparación de información y su análisis y busca los cambios en los negocios.

Algunas de las tecnologias emergentes que se mencionan son: el uso de sensores en la producción, el código de barras en las ventas, control de inventarios, seguimiento de artículos, tecnología móvil, lápiz electrónico, sistemas-diagnósticos para el sistema de pruebas y el intercambio de datos, multimedia, inteligencia ambiental, reconocimiento de voz, etc.

\section{Reingenieria}

La definición es el repensamiento profundo y rediseño radical de los procesos del negocio con el fin de lograr una mejora espectacular en el desempeño.

La reingeniería debería aplicarse después de que los esfuerzos de mejoramiento continuo no hayan dado resultados o cuando la problemática sea realmente seria.

Cabe aclarar que el profesor Toscano hace una analogía entre lo que eran las anteriores medidas recomendadas: una especie de medicina preventiva y en todo caso de cura, mientras que la reingeniería venía a ser una especie de cirugía y manifestaba que se debería hacer reingeniería cuando el proceso existente fuera un impedimento para la conducción del negocio o cuando existieran graves ineficiencias siendo su aplicación indispensable para recuperar la paridad y la competitividad en forma rápida para así obtener ventaja.

No podrá hacerse reingeniería si no se definen los procesos, se hace uso de la computación adecuada, se le da poder para que el empleado tome sus propias decisiones (empowerment); es decir, éste dar poder significa ampliar sus posibilidades, buscar la aplicación de la mentalidad del subordinado que participe, dar una educación adecuada y permitirle que tenga acceso a la información necesaria para su gestión. 


\section{Cultura organizacional}

La cultura organizacional se define como el conjunto de valores y creencias, normas y comportamientos, premisas y reglas no escritas compartidas por todos los miembros de una organización, que han sido adquiridas a través del tiempo. Esto incluye la forma y manera en el uso de las comunicaciones.

Los aspectos que constituyen esta cultura están referidos al ejercicio adecuado de la autoridad que requiere, del que lo ejerce, una capacidad de ver en forma amplia y a futuro, de participar en forma activa y comunicarse, de despertar confianza y ofrecerla a sus subordinados y un aspecto muy importante que guarda relación con esta última característica y es que se le debe dar al trabajador capacidad de decisión.

Se debe buscar la forma de trabajar en equipos que incrementen los resultados por el uso conjunto de talentos, compartiendo con ellos un propósito común, buscando responsabilidad mutua, evitando organizaciones demasiado dosificadas y estructuradas rígidamente.

Debe desarrollarse un sentimiento societario entre los trabajadores, directivos y dueños por medio del logro de beneficios mutuos, de la unión, de una actitud mental positiva, de la utilización adecuada de recursos, del conocimiento compartido y de involucrarse en diagnosis, acciones y resultados.

Por último se recomienda la evaluación objetiva del concepto de cultura organizacional considerando las variables internas y externas, en función de obtener resultados.

\section{LA GERENCIA Y EL COMUNICADOR ORGANIZACIONAL}

La gerencia no puede realizarse en forma permanente y exitosa por una sola persona o por un grupo limitado de personas. Debe buscarse la participación de todos los que integran los equipos humanos de la organización mediante la comunicación sistemáticamente establecida. Es necesario señalar participativamente la visión que se tiene a futuro, definir la misión genérica que se persigue y buscar la esquematización de estructuras flexibles que permitan mayor participación y comunicación que hagan posible la autoevaluación de las organizaciones, teniendo presente los esquemas básicos de las ciencias administrativas. Vale decir, 
cómo se programan y controlan participativamente las actividades, cómo se permite que fluya la información hacia aquéllos que coadyuvan a la evaluación, diseño de estrategias y programas, y que operan integradamente sobre la base del análisis de costos, presupuestos y búsqueda de márgenes de contribución al superávit o utilidades de la organización; cómo se mide y recompensa los esfuerzos para competir por medio de la justicia distributiva; cómo se dota adecuadamente de recursos físicos, materiales y tecnológicos a la institución y a los trabajadores; cómo se mantiene un espíritu de participación; cómo comunico a la comunidad mis intenciones y cumplo con éstas.

En suma, el ser humano y las comunicaciones son el núcleo fundamental en la dirección de las entidades. 
Bibliografía

Argyris, Chris

1964

Babbage, Charles

1832

Beckard, Charles

1993.

Como motivar el cambio en la organización. Bogotá: Editorial Norma S.A.

Bertananfly, Ludwig von 1976

Teoria general del sistema. Madrid: Fondo de Cultura Económica.

Blake R.R. [y] J.S. Mouton 1964

La parrilla gerencial. Clave para la toma de decisiones. Houston: Gulf Publishing Co.

Chiavenato, Idalberto 1995

Introducción a la teoría general de la organización. Bogotá: McGraw Hill Co.

Deming W. Edwards 1988

Fayol, Henri

1967

George, Claude

1972

Hermida, Jorge et al.

1992
Administración industrial y general.

Administración industrial y general. S.A.

Historia del pensamiento administrativo. Nueva York: Prentice Hall Inc.

Calidad total. Bogotá: Editorial Norma S.A.

Administración y estrategia. Bogotá: Ediciones Macchi. 
Herzberg, F., F. Mausner [y] B. Snyderman

1959

La motivación del trabajo. Nueva York: John Wiley and Sons Inc.

Kast F.E. [y] J.E. Rosenzweig

1970

Gerencia de las organizaciones. Un concepto de sistemas. Nueva York: McGraw Hill.

Likert, Rensis

1967

La organización humana: Su valor y gerencia. Nueva York: McGraw Hill.

Maquiavelo, Nicolás

1950

El principe. Nueva York: Random House.

McGregor, Douglas

1960

El lado humano de la empresa. Nueva York: McGraw Hill.

Mooney, James

1939

Los principios de la organización. Nueva York: Harper \& Bros.

Ouchi, William

1982

Teoría Z. Bogotá: Fondo Educativo Interamericano.

Porter, Michael

1980

Estrategia competitiva. Nueva York: MacMillan Inc. Free Press.

1985

Ventaja competitiva. Nueva York: MacMillan Inc. Free Press.

1990

La ventaja competitiva de las naciones. Nueva York: MacMillan Inc. Free Press.

Pozo Navarro, Fernando 1974

La dirección por sistemas. Madrid: Gráfica Carreras.

Reddin W.J.

1970

Eficacia gerencial. Nueva York.

Schein, Edgard

1965

Psicología organizacional. San Francisco: Jossey Bay Publishers

Simon, Herbert 1965

Conducta administrativa. Toronto: Collier MacMillan Canadá. 
Skinner, B.F.

1953

Ciencia y conducta humana. Nueva York: MacMillan Company Free Press

Smith, Adam

1980

La riqueza de las naciones. Londres: Adam Smith Int. Ltd.

Tannenbaum R., R. Weschler [y] Massarik

1961

Organización y liderazgo. Nueva York: McGraw Hill.

Taylor, Frederick 1967

Los principios de la administración científica. México: Herrero Hermanos Sucs. S.A.

Yip, George

1993

Gerencia estratégica y globalización. Nueva York: Niley. 